\title{
Aportes de la escuela en casa para niños y niñas con necesidades educativas especiales
}

Investigadora independiente, propietaria y tutora del Centro Educativo Alternativo ParuParo, San José, Costa Rica; sofia.chirino@gmail.com

Recibido: 24 de abril del 2019

Corregido: 04 de setiembre del 2019

Aceptado: 27 de setiembre del 2019

\begin{abstract}
Resumen
El presente articulo muestra parte de los resultados de una investigación cuyo propósito fue exponer las principales características de una modalidad didáctica que emerge en la educación primaria: la escuela en casa. Por ello en este texto se presenta en primera instancia la situación actual de esta modalidad educativa en Costa Rica, cómo se lleva a cabo y el tipo de apoyo que ofrece en casos específicos de niños y niñas con necesidades educativas especiales. Los datos que se discuten en este articulo se enmarcan en el enfoque cualitativo y se centra en el estudio de la vivencia de dos menores con necesidades educativas especiales, que habitan en la ciudad de San José y que estudian hace más de dos años bajo la modalidad de escuela en casa. Para ello, se realizaron observaciones en el centro donde reciben tutorías, se entrevistó a ambos progenitores y a la docente tutora. Entre las principales conclusiones que se exponen está el que la modalidad de Escuela en casa ha ofrecido a los dos menores observados buenas herramientas para un desarrollo integral, además de la formación esperada según los perfiles de salida de los Programas de Estudio del Ministerio de Educación Publica. A partir de este hallazgo se espera que esta discusión abra puertas a nuevos estudios que profundicen en el uso de esta modalidad para la educación primaria en el país.
\end{abstract}

Palabras clave: Educación primaria; escuela en casa; necesidades educativas especiales; apoyo pedagógico; tutoría; enseñanza personalizada.

\section{Abstract \\ Homeschool contributions for children with special educational needs}

This article shows part of the results of an investigation whose purpose was to expose the main characteristics of a didactic modality that emerges in primary education: homeschool. Therefore, in this text the current situation of this educational modality in Costa Rica is presented in the first instance, how it is carried out and the type of support it offers in specific cases of children with special educational needs. The data discussed in this article is framed in the qualitative approach and focuses on the study of the experience of two children with special educational needs, who live in the city of San José and who studied more than two years ago under the modality of home school. For this, observations were made in the center where they receive tutorials, both parents and the tutor were interviewed. Among the main conclusions set out is that the modality of homeschool has offered the two minors observed good tools for integral development, in addition to the expected training according to the output profiles of the Study Programs of the Ministry of Public Education. Based on this finding, this discussion is expected to open doors to new studies that deepen the use of this modality for primary education in the country.

Key words: Primary education; homeschool; special educational needs; pedagogical support; tutorial; personalized teaching. 


\section{INTRODUCCIÓN}

Este artículo retoma y discute parte de los principales hallazgos de la investigación titulada Análisis del apoyo pedagógico que se brinda dentro de la modalidad educativa "Escuela en Casa" a un estudiante de primer grado y a uno de quinto grado con Necesidades Educativas Especiales, quienes habitan en la provincia de San José y que son atendidos por personas tutoras, sus padres y madres durante el primer semestre del 2018. Hacia un modelo más inclusivo de educación en Costa Rica, efectuado por Chirino (2018)

El estudio tiene una relación directa con un fenómeno social y educativo conocido como Escuela en Casa (o Homeschooling como se denomina en inglés), esta modalidad didáctica en la educación primaria se ha desarrollado en diversos países de Europa y Norteamérica, y poco a poco ha llegado hasta países de América Latina, incluido Costa Rica, trayendo diversas experiencias para la educación costarricense.

La investigación realizada por Chirino (2018) y cuyos resultados se retoman en este artículo, se concretan exclusivamente en los beneficios que esta metodología ofrecería a niños y niñas con alguna necesidad educativa especial.

La escogencia de esta temática se basa en la hipótesis de que la modalidad de escuela en casa incide positivamente en infantes con necesidades educativas especiales, logrando aprendizajes significativos de diversas destrezas y habilidades cognitivas y sociales, que por diversas circunstancias que se dan en las escuelas regulares, no siempre se pueden atender oportunamente.

El estudio de Chirino (2018) se centró en la experiencia de dos niños que estudian dentro de este tipo de educación desde hace dos años, bajo la tutoría de una docente (quien tiene más de 25 años de desarrollar esta modalidad en el país, y tiene vasta experiencia con niños y niñas con necesidades educativas especiales), más el apoyo de los padres y madres de ambos menores quienes formaron parte de la investigación.

\section{Escuela en casa en el contexto educativo costarricense}

Actualmente existe una cantidad de estudiantes de primaria que están siendo diagnosticados con problemas de atención, hiperactividad y otros, para quienes el padre y la madre de familia buscan alternativas que les permita continuar dentro del sistema educativo, pero desde otra modalidad didáctica.

Este tipo de iniciativas encontrarían alguna forma de apoyo institucional, pues el Programa de Educación Abierta del Ministerio de Educación Pública, ofrece una serie de opciones que acreditan los estudios de I, II y III Ciclo, para aquellas perdonas que deciden salir del sistema o que han sido formalmente rechazados, para que puedan continuar sus estudios.

Las personas que optan por la modalidad de educación abierta para sus hijos e hijas, en la mayoría de casos (no existen estadísticas sobre esto) realizan matricula en escuelas extranjeras que son también conocidas como "escuelas sombrilla" y ofrecen la modalidad de educación a distancia para familias que practican escuela en casa.

Cabe resaltar que estos centros están debidamente acreditados en sus respectivos países, se encargan de vigilar el progreso del estudiantado y certifican sus estudios con notas reconocidas internacionalmente, para que quienes así lo deseen, puedan integrarse de nuevo a una institución publica o privada costarricense o de otro país.

El Ministerio de Educación acredita la formación de estas personas mediante distintas modalidades, sin embargo, la que más se utiliza para los estudios de I y II ciclos consiste en una prueba escrita que evalúa 
los aprendizajes por cada materia básica cuando la o el estudiante se encuentra listo y tiene el manejo necesario de los temas del curriculum.

Posteriormente, para acreditar los estudios de secundaria, los estudiantes hacen los exámenes de bachillerato por madurez al cumplir los 18 años o bien pueden aprobar secundaria de otras maneras, entre esas, la de realizar exámenes por cada materia básica cada año de secundaria para ir acreditando cada nivel por separado.

Es especialmente significativo estudiar el periodo de Escuela en casa, durante l y ll ciclo, pues en el país la mayoría de familias que utilizan esta modalidad educativa, opta luego porque sus hijos e hijas se incorporen al sistema educativo tradicional durante la etapa de secundaria (no existen datos sobre esto). Por esta razón las tutorías privadas que se brindan al estudiantado deben apegarse a los perfiles, objetivos y los planes de estudio del Ministerio de Educación Pública.

Por otra parte, en el IV Informe del Estado de la Educación (Programa Estado de la Nación, 2013) se indica que "un $77,6 \%$ de las y los docentes, refieren las necesidades educativas especiales como carencias de los y las estudiantes y solo un $12,8 \%$ las asocia con condiciones del entorno escolar" (p.15). Esta estadística sugiere cuestionamientos sobre el tipo de atención que se brinda a un estudiante con adecuación curricular en el aula.

Sobre esto, el IV Informe del Estado de la Educación (Programa Estado de la Nación 2013) dice que "el sistema escolar que conocemos en términos de factores físicos, aspectos curriculares, expectativas y estilos de profesorado y roles directivos tendrá que cambiar" (p.23). Este cambio de sistema se torna necesario, ya que se parte del hecho de que el ser humano es esencialmente diverso y la educación debe responder a esa diversidad. Sobre todo, en el caso particular de estudiantes que son diagnosticados con déficit atencional u otras condiciones, ya que muchos encuentran dificultad para adaptarse a la manera de evaluación y en casos optan por desertar.

Se dice que para llevar a cabo con éxito las adecuaciones curriculares en la dinámica del aula de primaria, es necesario crear un ambiente inclusivo, esto quiere decir, abrir un espacio en el que se reconozcan las diferencias entre los estudiantes como condiciones propias y naturales de todo ser humano. Sobre este abordaje de las Necesidades Educativas Especiales, Aguilar (2008) menciona que:

... la mayor confusión en el concepto de necesidades educativas especiales radica en considerarla una categoría diagnóstica y no un abordaje educativo [...] donde el interés principal no se centra en etiquetar a un (a) estudiante determinado, sino en determinar cuales son las barreras que, a partir de la propuesta curricular, la oferta educativa y las practicas educativas, están impidiendo que los (as) alumno (as) aprendan." (p.72)

De acuerdo con este autor la mayoría del estudiantado diagnosticado con algún tipo de Necesidad Educativa Especial no recibe una atención adecuada durante el trabajo en clase, pues únicamente se utiliza ese diagnóstico para otorgarle una "categoría especial" a la persona que justifique el porqué de su rendimiento escolar.

De esta situación se pueden derivar diversas problemáticas, pero la más significativa es el declive en el rendimiento y autoestima de los estudiantes, quienes después de un tiempo pueden asumir que no son capaces y que cualquier reto está muy lejano a ser superado. Por ello, en este articulo se discute en torno a qué pasa con familias que han optado por la modalidad de Escuela en casa, porque desertaron del sistema educativo tradicional con la intención de ofrecer una atención escolar mas personalizada a sus hijas e hijos. 
Según lo antes anotado, los datos que aquí se abordan provienen de la investigación efectuada por Chirino (2018), a través de los cuales se buscó responder ¿cómo es el apoyo pedagógico que se brinda dentro de la modalidad educativa Escuela en casa a dos estudiantes con necesidades educativas especiales, quienes son atendidos por una persona tutora y por sus padres y madres?

\section{Modalidad Escuela en casa}

Esta modalidad ofrece una educación de calidad respetando los ritmos, interés y necesidades especificas de cada estudiante, y se ha posicionado en Costa Rica como un método educativo alternativo que ofrece diversas ventajas al ser utilizado en determinadas circunstancias donde se requiere una atención escolar más personalizada.

Es en esta perspectiva que ciertos modelos han informado e incluso inspirado los métodos de educación en casa, algunos de ellos como metodologías educativas en si mismas.

Por ejemplo, los sistemas Waldorf, Montessori y el denominado Reggio Emilia, son propuestas educativas y a su vez, en muchos casos, metodologías utilizadas en sistemas alternativos como el Cyber Charter School, el Unschooling u otros enfoques educativos que mezclan lo presencial y lo virtual, pero el sistema que interesa para efectos de este estudio es el de la Escuela en casa.

En el país, la Escuela en casa se da principalmente cuando niñas o niños tienen alguna necesidad específica que no se ve atendida en el sistema tradicional, en muy pocas ocasiones se trata de estudiantes que nunca han estado en un sistema formal.

Esta modalidad educativa permite que padres, madres o encargados de niñas y niños puedan involucrarse en la toma de decisiones sobre los contenidos a estudiar, el acomodo del tiempo de estudio y el descanso durante la semana, las actividades extracurriculares, entre otros aspectos.

Las personas encargadas pueden escoger el tipo de Escuela en casa que quieren llevar a cabo, pues actualmente existen distintos programas, ya sea en línea o por medio de paquetes de libros que facilitan la organización de los temas, las actividades y sus respectivas evaluaciones. Sin embargo, también hay tutores y centros educativos informales que ofrecen el servicio de clases particulares, teniendo como ventaja que los estudiantes participantes pueden socializar con otras personas durante las lecciones.

Como un antecedente relevante de la Escuela en casa se suele mencionar el pensamiento de Iván Illich (1973). Este pensador destacó en el XX por sus importantes observaciones sobre la educación (Deschooling society), la tecnología (Tools for Conviviability), la relación entre la energía y el desarrollo (Energy and Equity) y la medicina, entre otros temas significativos para el pensamiento occidental.

Illich (1973) propuso un debate sobre la corrupción de las instituciones, corrupción que muchas veces hace que estas no alcancen su propósito, sino mas bien retrocedan de los objetivos originalmente planteados. Observó estos procesos especialmente en la escuela tradicional y en los sistemas médicos. Su observación sobre la escuela fue una parte muy importante e influyente de su pensamiento. En su libro, Deschooling Society (Illich, 1973), hizo un análisis de los efectos negativos de la escuela y desarrolló una critica del monopolio dominante de las tecnologías de la educación.

La observación de Illich (1973) va dirigida, principalmente, contra esos recursos económicos provistos por el Gobierno Federal a las escuelas y cómo, a pesar de esfuerzo fiscal, el estudiantado no mejoraba en su formación escolar. La intuición de llich va dirigida, entonces, a que no es la cantidad de dinero que se invierte, sino como institucionalmente el sistema esta organizado para que no se alcancen los mejores objetivos con las poblaciones escolares. 
Según ese autor, los niños pobres seguirán teniendo dificultades a pesar de asistir a escuelas donde hay condiciones "igualitarias", pues habrá factores diversos que inciden en los mejores resultados de los niños y niñas de grupos sociales mas aventajados, como los son, la mejor escolaridad de los padres, mejores conversaciones y actividades extracurriculares más enriquecedoras, más libros disponibles en casa, mejores vacaciones y en general mejor tiempo de calidad.

Esta relación entre la educación "escolarizada" y la que puede ser provista de otra manera, resulta hoy una preocupación significativa en diversos países del orbe y constituye un debate que apunta hacia el deber del Estado de proveer una educación gratuita de calidad, y las posibilidades de sistemas educativos alternativos como la Escuela en casa.

Actualmente existe legislación que permite la modalidad de Escuela en casa en países como Portugal, Francia, Inglaterra, Irlanda, Bélgica y Dinamarca (Calzada 2013).

Según Soberanes y Trejo (2011), junto a la crisis institucional del Siglo XX, y la desconfianza respecto de sistemas institucionalizados como la educación, hubo un retorno de la Escuela en casa. Bajo el lema de que era mejor educar a los hijos en el seno del hogar, preservando la propia identidad y los valores, así como con la idea de que de esa forma era posible formar hijos mas autónomos y mas independientes, la Escuela en casa cobró auge en el contexto social de los Estados Unidos, especialmente en la década de los años 60.

En el Reino Unido se puso en vigencia la ley de Educación de 1996 (Education Act) que permitió la enseñanza en el hogar, pero no dejándola a completa libertad de padres y madres, sino con ciertos controles institucionales para garantizar una educación adecuada a las necesidades, aptitudes, habilidades y la edad del estudiantado.

En España, la escuela en casa inició con cuarenta familias en la década de los años noventa y se crearon diversas asociaciones civiles, por ejemplo: la Asociación por la Libre Educación (ALE), Educar en Casa, Crecer en Libertad y Educación en Familia.

La práctica en Costa Rica no esta lejana a las situaciones antes mencionadas, ya que son precisamente familias que buscan una mejor atención para sus hijas e hijos las que han puesto en practica esta modalidad, personas que por distintas razones no quedaron satisfechas con el tipo de educación que estaban recibiendo, o que esperan proveer una mejor calidad de enseñanza que la ofrecida por la escuela tradicional.

La Escuela en casa procura el empoderamiento de padres y madres en el proceso educativo de sus hijas e hijos. Los importante, con todo, es la forma en que se puede organizar el estudio individual, no hay horarios pre-establecidos más allá de los que la propia familia se imponga, no hay objetivos curriculares marcados por una escuela. Los niños, niñas (padres y madres) no tienen la presión de saber qué necesariamente deben aprender en determinada materia o en determinado plazo. No hay pruebas escritas.

En ese ambiente, la posible atención de Necesidades Educativas Especiales (NEE) se observa propicia y hace aumentar la esperanza de un acercamiento que compromete a las personas que componen el hogar del estudiante, lo cual no siempre da en los sistemas educativos tradicionales que no suelen estar adecuadamente preparados para atender al estudiantado con NEE, no sólo desde la perspectiva del diagnostico y de la definición e implementación de las estrategias educativas, sino también desde la óptica de la adaptación individual del currículo, de las metodologías y de la vinculación de padres y madres.

Lo más frecuente es que haya una mezcla de sistemas metodológicos, entre los más utilizados se suele mencionar Montessori, el método clásico (que incluye Trívium y Quadrivium), la educación libre, el de las inteligencias múltiples, el de Waldorf. Los padres que escogen esta educación en casa, suelen frecuentar bibliotecas publicas, editoriales, internet y materiales hechos específicamente para la educación 
en casa. Esto quiere decir que el esfuerzo metodológico es variado y las fuentes que lo respaldan variadas también.

Al respecto, Bull, Brooking y Campell (2008) mencionan que:

...podemos encontrar otros con diferentes metodologías y enfoques pedagógicos, que son muchos y muy variados y que el homeschooling manifiesta; por ejemplo, las competencias parentales que se desarrollan para apoyar el aprendizaje y la combinación de metodologías mas estructuradas y menos estructuradas o de enfoques mixtos, más dirigidos o más libres (p. 19)

Esta flexibilidad para poder armar un plan de estudios según lo que el estudiante necesite y las personas encargadas consideren mejor, es un aspecto que ya por si solo presenta una de las grandes ventajas de la modalidad de Escuela en casa para atender las NEE, ya que en muchos casos los requerimientos son mas específicos que otros y el poder ofrecer un servicio a la medida es sumamente valioso.

\section{METODOLOGÍA}

La investigación que fundamenta este articulo se desarrolló desde el enfoque cualitativo, ya que buscó responder problemas que se derivan de preguntas tales como: "qué es lo que sucede" o "cómo sucede". Este tipo de interrogantes son las típicas del ámbito educativo, cuando se estudia qué sucede en un salón de clases donde interactúan maestros y estudiantes, o donde se evalúa el proceso de enseñanza-aprendizaje desde la perspectiva de las personas involucradas.

Precisamente ese fue el entorno de esta investigación, donde la observación de los procesos de enseñanza y la superación de las dificultades por parte del estudiantado con capacidades educativas diferentes, puede generar conocimiento valioso para comprender las condiciones en que se producen los procesos de la clase y en el hogar.

El tipo de investigación es fenomenológico, en cuanto y tanto como lo menciona Guardián (2007): “El método fenomenológico respeta, por completo, el relato que hace la persona de sus propias vivencias. Se centra en el estudio de las realidades vividas o vivencias, generalmente, poco comunicables" (p. 156)

La investigación que se realizó se ubicó en la provincia de San José, específicamente en el lugar donde se llevan a cabo las tutorías con los estudiantes que participaron de esta investigación, durante el primer semestre del año 2018.

Cabe resaltar que el centro visitado atiende a niños y niñas desde los 2 años hasta estudiantes adolescentes que cursan bachillerato. Cuenta con especialistas en distintas áreas como, por ejemplo: psicopedagogas, psicólogas y docentes de preescolar y primaria, esto, para poder brindar un servicio completo a los estudiantes, ya que defienden que el éxito académico va de la mano del bienestar emocional.

La profesional docente que colaboró posee título en docencia bilingüe y una especialidad en psicopedagogía, además es madre de familia y tutora de varios estudiantes que estudian bajo la modalidad de Escuela en casa. Tiene una experiencia de más de 25 años en el campo de esta metodología y desde entonces ha graduado a muchos estudiantes que estudian en casa. Este estudio se concretó en esta única experiencia, ya que cuenta también con la especialidad en atención a las necesidades educativas especiales.

Los estudiantes participantes fueron dos niños de segundo y quinto grado quienes estudian bajo la modalidad de escuela en casa con la tutora anteriormente mencionada. 
En el caso de uno de ellos, el niño cuya edad es 8 años ha sido diagnosticado en el espectro autista sin embargo ninguno de sus diagnósticos han sido bien justificados ya que se contradicen mucho uno y el otro, hay doctores que han dicho que el niño no tiene una NEE importante y otros afirman que tiene un trastorno de bipolaridad, causando que el niño por momentos actúe de forma sumamente violenta. Tiene dos años de estar estudiando bajo la modalidad de escuela en casa, e ingresó después de haber estado en primer grado en una escuela tradicional seis meses.

El otro caso se trata de un niño de 11 años con trastorno de hiperactividad con déficit atencional y va a cumplir casi dos años de haberse retirado del sistema educativo tradicional. Padece de la enfermedad de Crohn, condición que le ha afectado bastante y ha tenido que pasar por largos periodos en el hospital o en reposo en su casa.

Ambos comenzaron a estudiar bajo esta modalidad despues de varios intentos fallidos en el sistema tradicional, el cual en distintas ocasiones recomendaron que lo mejor era

Las técnicas para recolectar información en la investigación fueron la entrevista a profundidad y la guía de observación. Se llevó a cabo una entrevista a profundidad para la persona tutora y otra para los padres y madres de los estudiantes.

Las entrevistas buscaban indagar sobre estrategias de aprendizaje, apoyo pedagógico, planificación, evaluación, socialización y el trabajo en equipo que se lleva a cabo dentro de la modalidad de Escuela en casa.

La guía de observación que se utilizó, contó con una lista de indicadores sobre aspectos importantes a observar. Fue especifica porque permitió registrar con claridad las características que le interesaba estudiar a la investigadora en las distintas categorías, sin embargo, también fue abierta pues permitió introducir otros aspectos de la modalidad de Escuela en casa que se consideraron de importancia para la investigación.

La observación se llevó a cabo en el lugar que se imparten las tutorías de los estudiantes, razón por la cual se pudo observar las practica educativa en sus distintas dimensiones. Se realizaron en total 6 visitas de dos horas aproximadamente, las cuales se caracterizaron por ser no participativas, pues la persona observadora en ningún momento se involucró en las dinámicas. Se pudo examinar con detalle tanto del espacio en que se desarrollaron las tutorías, así como de los materiales que los niños utilizaban.

\section{RESULTADOS}

En el país, en algunos casos la personas que estudian bajo la modalidad de escuela en casa, en ocasiones, acuden a centros donde son atendidos por tutores licenciados en educación. En estos espacios pueden compartir con otras personas de su edad y recibir tutorías completamente individualizadas, ya que se adaptan al ritmo y las necesidades de cada estudiante en particular sin descuidar el aspecto de la socialización.

La experiencia de tutorías observada para efectos de la investigación da lugar a este artículo, se caracterizó por contar con grupos multiedad, en los cuales se estudiaba de distintas formas: en ocasiones cada estudiante trabajaba individualmente, pero acompañados por la tutora; en otras, se ocupaban proyectos colectivos y todos compartían y estudiaban el mismo tema. Además, se pudo observar que también contaban con tiempo para juego libre o para realizar alguna manualidad.

De manera general, durante las visitas al lugar donde se realizan las tutorías, se pudo observar que el estudiantado tiene un sentido de responsabilidad distinto al que se acostumbra observar en las escuelas de sistema tradicional. El poder contar con la flexibilidad de elegir el trabajo diario según el criterio de 
cada estudiante, les ayuda a tomar conciencia sobre la labor que deben realizar y lo que se espera de ellos, adicionalmente este sistema les permite interesarse genuinamente por lo que deciden hacer. Por ejemplo, si se enfocan primero en la asignatura de Ciencias y eligieron el tema del sistema digestivo, entonces la atención que le van a prestar es significativa y especial, por el simple hecho de que fue libremente escogido Las niñas y los niños participan activamente durante la lección y se aseguran de terminar bien su trabajo para después poder disponer su tiempo en otras actividades no necesariamente académicas, puede ser una manualidad, jugar, comer o simplemente conversar con los compañeros.

En el hogar, los padres y las madres también ofrecen apoyo, entre otras maneras, al decorar la casa con material didáctico llamativo, manteniendo así, la misma línea de trabajo que la persona tutora. De esta manera se enriquecen constantemente los aprendizajes. Adicionalmente se les recomienda a las familias que el o la estudiante realice actividades extracurriculares, entre las cuales se destacan clases de música, baile, natación, pintura, karate, taekwondo, idiomas, entre otras. Las anteriormente mencionadas son las más frecuentadas.

Parte de los apoyos que también se les brindan a ambos estudiantes por parte de las familias, consiste en facilitarles experiencias educativas lúdicas, entre las cuales se pueden mencionar: excursiones a museos, paseos a parques nacionales, obras de teatro y otra variedad de actividades culturales y sociales valiosas. Esto permite concluir que el compromiso de los encargados y de otros familiares es bastante alto, en pocas palabras, son ellos los responsables de perpetuar y enriquecer los conocimientos que ellos obtienen durante las tutorías.

Por otro lado, la forma de evaluación de los aprendizajes es mas libre: se evalúa a través de conversaciones y de observación constante de los avances de los niños. Los padres y madres, quienes de antemano han entendido y accedido a esa manera de evaluación, no exigen exámenes formales, sino que contribuyen en el proceso preguntando y conversando con los niños sobre los aprendizajes que obtienen en las tutorías y los temas que llaman de manera especial su atención.

La libertad y flexibilidad de este tipo de evaluación interesa por igual a padres y madres como a los estudiantes, pues reduce el estrés previo a una prueba escrita (lo cual incluye la preparación para resolverla y la tensión de los encargados porque los estudiantes logren entender y memorizar todos los contenidos por evaluar).
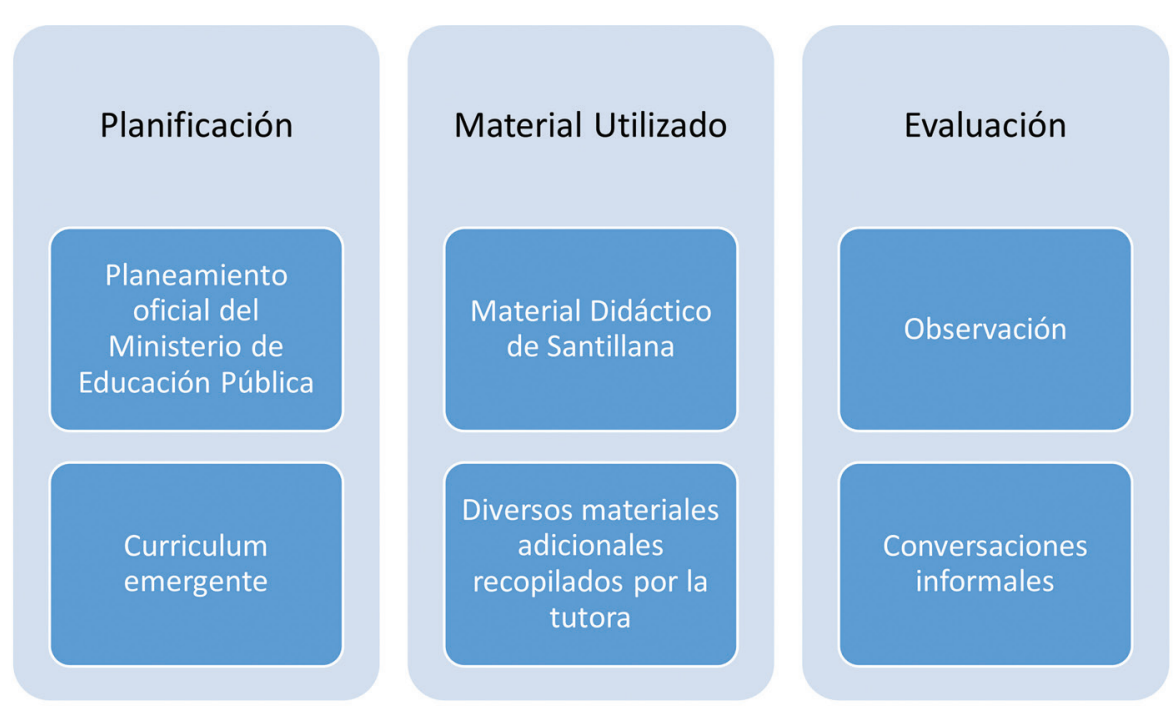

Figura 1. Características de la planificación, evaluación y material utilizado. Fuente: Elaboración propia a partir de Chirino (2018). 
En la siguiente figura se pretende mostrar de manera concisa las características de la planificación, evaluación y el material utilizado en las tutorías, según las observaciones efectuadas.

Haciendo referencia a los recursos antes mencionados cabe resaltar que durante las observaciones se pudo constatar que el trabajo diario estaba compuesto por un balance armonioso entre los libros utilizados a nivel nacional por las escuelas, el material adicional proveído por la tutora y las actividades que los mismos estudiantes proponían. La evaluación es, efectivamente, constante ya que el trabajo que se realiza es guiado por la tutora, quien tiene la oportunidad de determinar si es necesario un refuerzo o si es posible que el estudiante continúe trabajando y avanzando con otros temas.

Los padres y madres que participaron en las entrevistas subrayaron la relevancia del acompañamiento que realizan, manifiestan que esto desarrolla interés por el proceso educativo de sus hijos. Además, mencionaron que intercalan otras actividades cotidianas del hogar lo cual, al mismo tiempo, contribuye a establecer otras formas de interacción y vínculos entre la familia, pues no solo se atienda la parte académica de los estudiantes, sino que se logra desarrollar otras habilidades como el cuido personal y del entorno y el aseo personal e igualmente del hogar. Al involucrarlos en las tareas de la casa los infantes logarían mayor madurez y sentido de responsabilidad por su entorno.

Es relevante destacar que el tipo de evaluación cualitativa llevada a cabo por la tutora, la convierte simultáneamente en una docente-investigadora que cumple un papel significativo en la formación integral de los niños, pues se encuentra en constante observación de sus características y necesidades para así realizar los cambios que sean necesarios en la forma de enseñar según corresponda y de esta manera comunicarlo a las familias para que sepan también cómo enfocarse y brindar apoyo desde el hogar.

Sobre esta forma de evaluación la tutora expresa lo siguiente:

(...la evaluación) Es por observación, y que ellos puedan resolver independientemente un ejercicio. El programa para nosotros tiene como fin ultimo el autocontrol, que la persona pueda autorregularse de manera en que pueda tomar decisiones, llegar, sentarse y trabajar o decidir si quieren ir allá o decir hoy no estoy para Mate, etc.

Continúa diciendo:

...entonces libera del estrés, y emocionalmente hablando, eso fortalece el proceso académico. Entonces yo, digamos, desde la parte psicopedagógica, se supone que tengo que tener estable todo lo emocional para que lo académico funcione. Entonces, el proceso de evaluación a través de la observación ya adelanta ese trabajo porque le permite trabajar sin el estrés de que vas a ser evaluado.

Por último, la tutora y su equipo de trabajo (constituido por docentes de primaria, asistentes de primaria y prescolar, psicólogos y psicopedagogos) se preocupan por brindar constantemente charlas y capacitaciones para los familiares y encargados, así como para el público en general, con el fin de apoyar en temas importantes como el manejo de límites, las estrategias de aprendizaje según distintas necesidades y consejos para hacer de esta modalidad una mas efectiva para todos los estudiantes.

Respecto al tema de la socialización de los niños, hay preconcepciones sobre la escuela en casa como la idea de que esta modalidad educativa aísla e impide procesos ricos de interacción entre otros niños de edad parecida.

Sin embargo, en las observaciones se identificó una realidad distinta, pues, pudo verse que no se apartaba a los niños de la interacción social, todo lo contrario, se propiciaban medios para su integración 
y generar un entorno que les diera confianza para ser ellos mismos y relacionarse con los demás a su propio ritmo y manera.

Una de las madres participantes en la investigación explicó que:

A ellos les cuesta mucho la socialización entonces entre más personas ellos tengan alrededor, más se inhiben. Como son poquitos entonces el tiene la oportunidad de ir como uno por uno conociendo y acoplarse poquito a poco. A su ritmo. Tiene como más chance de socializar bien.

Así, según lo observado, la socialización y el trabajo colaborativo en esta modalidad educativa ofreció a los estudiantes una vivencia social normal y con menos presiones: les permitía socializar respetando su individualidad facilitando, además, que los niños tuvieran mas tiempo para compartir con sus amigos, conversar, jugar, hacer alguna manualidad o trabajar en proyectos de interés común.

A continuación, se presenta un grafico que ayuda a ilustrarse de mejor manera las ventajas que tiene el tipo de socialización que se da en entornos de escuela en casa.

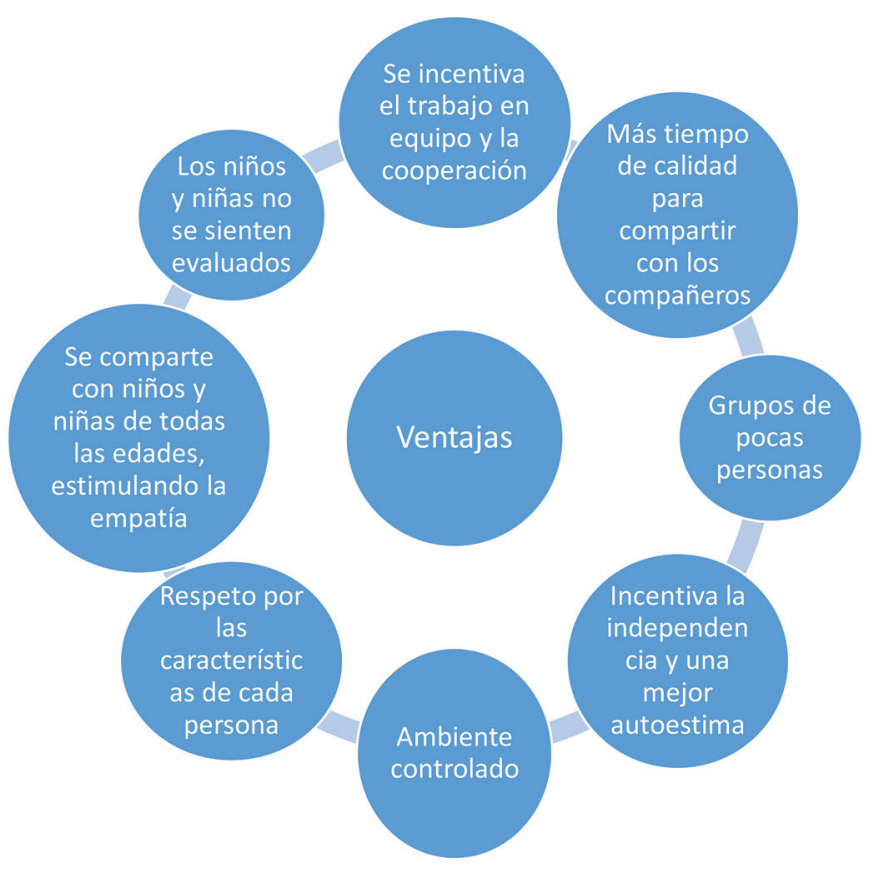

Figura 2. Ventajas del tipo de socialización que se lleva a cabo en la modalidad de escuela en casa. Fuente: Elaboración propia a partir de Chirino (2018).

Se rescata que en un entorno como el observado en efecto los estudiantes pueden socializar de manera libre, sin presiones y teniendo la oportunidad de mostrar su personalidad en un ambiente de respeto y amor.

Como en la modalidad de Escuela en casa el trabajo es flexible y no se castiga en la calificación al estudiante que hable con otro compañero durante clases, por ejemplo, favorece un ambiente que invita a la comunicación y a la socialización en todo momento. No dependen del tiempo de recreo para poder compartir con sus amigos sobre los temas que les interesan y gustan y por ende esta modalidad educativa demostró poder ofrecer una forma de socialización beneficiosa para los dos estudiantes participantes. 


\section{CONCLUSIONES}

Las observaciones de este estudio muestran como en el caso de los dos estudiantes que participaron en la investigación, se presentan opciones novedosas para niños y niñas con Necesidades educativas especiales y esto no puede quedar relegado a entornos familiares y las personas tutoras, que no pueden dejar de pensar que trabajan como si fueran una subcultura o que realizan trabajo en una zona legal gris, ya que por muchos la modalidad es vista casi como algo que atenta contra el bienestar de los menores.

Es necesario recalcar que, en muchos de los casos de estudiantes con alguna necesidad educativa especial, este tipo de estrategias en definitiva les ayudan de manera más eficiente para lograr los objetivos de los cursos, ya que muchas veces la repetición, la memoria o copiar de la pizarra no son estrategias adecuadas para el tipo de aprendizaje de los niños y niñas.

Metodologías como las mencionadas en éste artículo defienden la necesidad de involucrar al niño en su proceso educativo para que este sea significativo, y para lograr este involucramiento hay que tomar en cuenta que primero la persona debe sentirse motivada y citando las palabras de Martínez Salanova en el trabajo de Villaroel (2012):

Es responsabilidad del adulto identificar las capacidades, necesidades e intereses del infante y abordarlas a partir de metodologías basadas en el amor que conlleva al encuentro afectivo, a la creación de vínculos como elementos básicos para la construcción de la propia identidad y la convivencia con los demás (p.81).

Estas propuestas pretenden superar la escuela que se rige bajo esquemas didácticos y curriculares rígidos y desde la lógica adultocéntrica y más bien buscan dar paso a lo emergente y espontáneo que surge en el momento de las clases en donde se estimula la fantasía, la innovación y, sobre todo, los vínculos que sostienen, que aportan y que transforman al estudiante, entre ellos el vínculo con el maestro, sus familiares y sus compañeros.

Al exponerse a actividades más dinámicas, como la construcción de un sistema del cuerpo humano, el estudiante tiene la oportunidad de introducirse al tema de una forma más atractiva e interesante y se puede lograr la motivación de manera más eficaz.

Como menciona la tutora, puede que muchos no se sientan atraídos por las actividades que ella planea, pero eso también se respeta. Ella rescata que este respeto al criterio de cada uno es clave para el éxito, ya que no sólo los enseña a conocerse mejor a ellos mismos, sino que también crea un mejor ambiente de trabajo y los estudiantes aprenden en carne propia que cada persona es distinta y que debe respetarse.

En las observaciones efectivamente se pudo constatar la libertad con que cuenta cada estudiante para cumplir su trabajo. Ellos pueden elegir en qué trabajar, cuándo y cómo, teniendo siempre presente que deben avanzar cada día un poco más. Llama poderosamente la atención la madurez con la que toman decisiones una vez que llegan a las tutorías.

Se tiende a pensar que tal libertad puede ser perjudicial para menores de edad, sin embargo, lo que se observó es que cada uno tiene sumamente claro lo que se espera de ellos, que el orden en que hagan las cosas no altera el resultado al final del día. El trabajo se realiza de manera fluida y con buena actitud, razón por la cual, aunque el rato sea breve, los niños aprenden.

Sobre este aspecto, Montessori (1986) menciona: "Llamamos a un individuo disciplinado cuando es dueño de sí mismo, y puede, por tanto, regular su propia conducta cuando resulte necesario para seguir alguna norma de la vida" (p.341). 
Es importante observar que en espacios de centros educativos la relación que existe entre el profesorado y los alumnos, sujeta a códigos de convivencia y disciplina, como mencionan Sotés y Urpí (2012), y en donde además, tanto los funcionarios como los estudiantes están limitados a espacios y tiempos determinados que no se pueden modificar, se contraponen con los lazos afectivos que se cultivan en espacios más individualizados como se observa en la modalidad de escuela en casa, en donde, tanto los estudiantes como los maestros pueden, en conjunto, tomar decisiones y armar el trabajo según convenga cada día en particular. Esto abre puertas para pensar que el éxito de los dos casos observados tiene que ver con la posibilidad de entablar una relación diferente y más cercana entre alumno, maestro y familia.

El maestro, en la modalidad de la escuela en casa tiene la posibilidad de acompañar a su estudiante e involucrarse en el proceso para ayudarle al niño o niña a lograr el aprendizaje deseado, esto a diferencia de los maestros en una escuela tradicional, quienes, por diversos motivos ajenos a ellos, no pueden sentarse a trabajar de la manera antes descrita con sus alumnos.

En el caso de niños y niñas con necesidades más específicas es innegable que este tipo de atención, mucho más personalizada, les brinda una buena oportunidad para aprender.

Cook, Bennett, Lane y Mataras (2013; quienes citan Duval et al, 1997), mencionan que el éxito en esta modalidad se debe probablemente al más alto grado de interacción de los niños con su padre-instructor y el mayor grado de atención individualizada que reciben en la educación en el hogar.

El poder mover el currículo de forma libre, especialmente en estos casos, es sumamente valioso, muchas veces, como menciona la tutora, es cuestión de poder darle al estudiante un poco de espacio para que vaya interiorizando los temas que quizá a principio de año se le dificulten, pero conforme va avanzando, termina el año tal y como los otros niños de primaria de su nivel.

Se pueden observar las similitudes existentes entre la forma de trabajo de la tutora y el tipo de apoyo que ella les brinda con las características de metodologías alternativas como Reggio Emilia, Montessori o Waldorf por mencionar algunas. La organización de los temas y objetivos, de lo más fácil a lo más complejo y de lo general a lo específico; la libertad que se le otorga al estudiante para decidir sobre su rutina diaria, la distribución del espacio, las actividades sensoriales llevadas a cabo y en general la dinámica que existe entre la familia, los estudiantes y la persona tutora, que se caracteriza por los lazos de confianza que se van trabajando gracias a la coordinación y comunicación que debe existir entre ambas partes para beneficiar el desarrollo integral de los niños.

La tutora defiende que este tipo de enseñanza permite que la persona cambie su idea de lo que es estudiar y aprender y logra después de un tiempo, o en algunos casos, poco tiempo, que el estudiante trabaje mejor y más independientemente.

Nuevamente se recalca que esta modalidad lo que parece presentar es una manera alternativa de abarcar el currículo sugerido por el Ministerio, lo cual podría responder muchas interrogantes ante la preocupación de diversas personas sobre estas modalidades alternativas de educación.

Según lo observado, este entorno es más como una familia, en la cual todos se preocupan por todos y tienen la oportunidad de llegar a conocerse bien, tanto entre niños y niñas como con los adultos tutores, lo cual realmente permite que el ambiente para el estudio sea apto para sentirse a gusto y trabajar con calma.

A modo de reflexión de cierre, en la educación primaria costarricense se hace cada vez más latente la necesidad de brindar mejor información sobre esta modalidad para poder ofrecer un apoyo real a las familias que están pasando por la transición escuela-casa, y para quienes aun no saben como ayudar a sus hijos e hijas con necesidades educativas especiales. 
Al no haber suficiente investigación, la modalidad permanece en una zona legal gris que de cierta manera genera desconfianza en los padres, madres, otros familiares y conocidos y termina desalentando a la población a intentar con sus hijos e hijas escuela en casa.

Los estudios generarían un ambiente de mas seguridad y confianza y se podrían comenzar a emplear regulaciones y otro tipo de apoyos por parte de las instituciones publicas del país, en especial el Ministerio de Educación y que así, mas familias puedan verse beneficiadas y enriquecerse entre sí.

\section{REFERENCIAS}

Aguilar, G., Bonilla, J., Cubero, L., Jimenez, C., Murillo, A. Montenegro, F... \& Vega, L. (2008). La Educación Especial en Costa Rica: Antecedentes, evolución, nuevas tendencias y desafíos. San José: Centro Nacional de Recursos para la Educación Inclusiva CENAREC.

Bull, A., Brooking, K., \& Campbell, R. (2008). Successful home-school partnerships. Report to the Ministry of Education by the New Zealand Council for Educational Research. Wellington: Ministry of Education.

Calzada, M. (2013). El homeschooling crece en España y busca la legalidad. Recuperado de https://fundacionmelior.org/archivado/el-homeschooling-crece-en-espana-y-busca-la-legalidad/

Cook, K., Bennett, K., Lane, J. \& Mataras, T. (2013). Beyond the Brick Walls: Homeschooling Students with Special Needs. Physical Disabilities: Education and Related Services, 32(2),98-111, https://doi.org/https://doi.org/10.14434/pders.v32i2.12997

Chirino, S. (2018). Análisis del apoyo pedagógico que se brinda dentro de la modalidad educativa "escuela en casa" a un estudiante de segundo grado y a uno de quinto grado con Necesidades Educativas Especiales, quienes habitan en la provincia de San José y que son atendidos por personas tutoras, sus padres y madres durante el primer semestre del 2018 (Trabajo de Graduación para optar por el grado de Licenciatura en Educación General Básica I y II Ciclos). Universidad Estatal a Distancia, San José, Costa Rica.

Gurdián, A. (2010). El paradigma cualitativo en la investigación socio-educativa. San José: EUCR.

Illich, I. (1973). Deschooling Society. Recuperado de http://www.arvindguptatoys.com/arvindgupta/ DESCHOOLING.pdf

Montessori, M. (1986). La mente absorbente del niño. México: Diana.

Programa Estado de la Nación. (2013). Cuarto Informe Estado de la Educación. San José: Programa Estado de la Nación.

Soberanes, J. \& Trejo, L. (2011). Educación escolarizada vs. Educación en Casa. Reflexiones sobre la Sentencia Homeschooling del Tribunal Constitucional Español. Cuestiones Constitucionales, Revista Mexicana de Derecho Constitucional. (25), julio-diciembre, 353-365.

Sotés, M. \& Urpí, C. (2012) Homeschooling y escuela flexible, nuevos enfoques. Navarra: Universidad de Navarra.

Valle, J. (2012). Enseñar en casa o en la escuela. La doctrina legal sobre el Homeschooling en España. Revista Perfiles Educativos, XXXIV(138), 167-182.

Verdugo, M. (1995). Personas con discapacidad. Madrid: Siglo XXI.

Villarroel, P. (2012). La construcción del conocimiento en la primera infancia. Sophia. Colección de Filosofía de la Educación. (13), 75-89. 